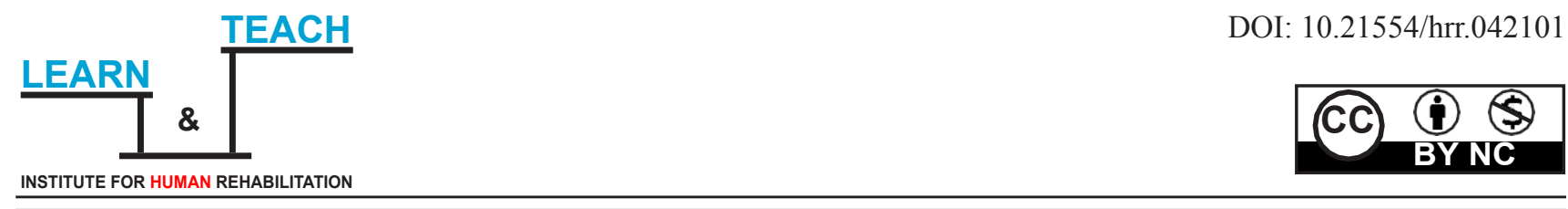

\title{
DYSPHAGIA IN PEOPLE WITH INTELLECTUAL DISABILITIES
}

Original scientific paper

\author{
Mirza Sitarevic ${ }^{1}$, Leila Begic ${ }^{2}$, Valentina Misanovic ${ }^{3}$ \\ ${ }^{1}$ PI „Elementary School Banovici“, „, Centre for the Development of Inclusive Practices “Banovici, Bosnia and Herzegovina \\ ${ }^{2}$ Faculty of Education and Rehabilitation, University of Tuzla, Bosnia and Herzegovina \\ ${ }^{3}$ PI ,Elementary School Dr. Boris Coric“ Kresevo, Bosnia and Herzegovina
}

Received: $2021 / 2 / 3$

Accepted: $2021 / 4 / 2$

\begin{abstract}
People with intellectual disabilities often face a number of disorders that can impair their health and even endanger their lives. One of these disorders is dysphagia, which is often unrecognized in this population. The main goal of the research was to determine the differences in the severity of dysphagia relation to the degree of intellectual disability in persons placed in institutional accommodation. The study involved 31 respondents diagnosed with intellectual disabilities. The results showed that people with a higher degree of intellectual disability have more pronounced dysphagia. Caregivers of people with intellectual disabilities should be aware of the presence of dysphagia in people with intellectual disabilities, and refer them to a speech-language pathologist in a timely manner with the goal of timely diagnosis and treatment to improve the overall health and quality of life of people of this population.
\end{abstract}

Keywords: swallowing disorder, degree of intellectual disability, institutional accommodation

\section{INTRODUCTION}

A human's ability to feed and swallow is a basic function essential to his/her health and well-being, providing him/her with the necessary nutrition and satisfaction. Swallowing involves the interaction of complex anatomical, neurological, and physiological systems. Even small changes in these systems such as swallowing time, deviations in anatomy or changes in swallowing physiology can have a profound impact on the whole process and significantly impair a person's quality of life (Lerner \& Tan, 2012, according to Begic \& Salihovic, 2018). Pathological changes in swallowing mechanisms lead to dysphagia, nutritional and social consequences (Kolundzic, 2012). Dysphagia is a symptom, and possibly a consequence of certain diseases.
It manifests as a disorder of bolus swallowing, with a frequent feeling of physical disturbance in its flow, regardless of its consistency, and can occur in any of the three phases of bolus flow from the oral cavity to the stomach (oral, pharyngeal and esophageal phase) (Begic \& Salihovic, 2018).

Dysphagia is a common occurrence in people with intellectual disabilities (Lindsay, 2011). According to the fifth edition of the Diagnostic and Statistical Manual of Mental Disorders (DSM-5), intellectual disabilities are disorders that occur during the development period, and include intellectual deficits and deficits of adaptive functioning in the conceptual, social and practical areas.

\footnotetext{
$\overline{\text { Correspondence to: }}$

Leila Begic, Faculty of Education and Rehabilitation Sciences, University of Tuzla

Univerzitetska 1 Street, 75000 Tuzla

Mob: +387 (0) 61502350

E-mail: leila.begic@untz.ba
} 
To diagnose intellectual disabilities, three criteria must be present, namely: Deficits of intellectual functions related to reasoning, problem solving, planning, abstract thinking, judgment, academic and experiential learning, and that they are confirmed by clinical assessment and individualized, standardized intelligence testing; Adaptive functioning deficits refer to difficulties in meeting developmental and sociocultural standards related to personal independence and social responsibility, and adaptive deficits limit functioning in one or more activities of everyday life such as communication, social participation or independent living in different environments such as home, work, school and community; The occurrence of intellectual and adaptive deficits is in the developmental period (American Psychiatric Association, 2013). The DSM-5 lists four levels/degrees of intellectual disabilities (mild, moderate, severe, and profound). However, levels/degrees are not determined based on IQ as in previous editions, but based on the level/ degree of adaptive functioning with the explanation that adaptive functioning is what determines the level/ degree of support required (American Psychiatric Association, 2013).

Swallowing and feeding disorders often occur in people with intellectual disabilities and can persist from birth to adulthood (Sheppard \& Hochman, 1988; Sheppard, 1991; Rogers \& et al., 1994; according to Sheppard, 2006). Dysphagia in people with intellectual disabilities has a high level/degree of complexity and variability, primarily affecting people with more pronounced cognitive impairments. The high prevalence of dysphagia affecting all stages of swallowing, and its progression with increasing age, indicate the need for a high level/degree of clinical caution (Sheppard, 2009).

Causes of dysphagia in a population of people with intellectual disabilities include oral-motor dysfunction, neurological abnormalities, psychiatric and behavioural disorders, gastroesophageal reflux, and musculoskeletal deformities (Walsh \& Feigelman, 1989, according to Sheppard, 1991). Some anatomical abnormalities may exacerbate dysphagia, such as the Gothic palate, while other abnormalities may be the primary cause of dysphagia, such as esophageal stenosis (Samuels \& Chadwick, 2006, according to Chadwick \& Jolliffe, 2009). The National Patient Safety Agency (NPSA) lists dysphagia as one of the five key risks to the safety of patients with intellectual disabilities (NPSA, 2004, according to Leslie, Crawford \& Wilkinson, 2008). Dysphagia has health implications that can be life threatening. These include poor nutritional status, dehydration, asphyxia, and aspiration that can lead to respiratory tract infections, which is one of the leading causes of death in the population of people with intellectual disabilities (Day \& Jancar, 1994; Helfrich-Miller et al., 1986, Kennedy et al., 1997, Rogers et al., 1994, Wood, 1994, according to Chadwick, Jolliffe, \& Goldbart, 2003). Dysphagia occurs more frequently in people with severe and profound cognitive impairment and severe physical disabilities, although it has been found that people who are mobile and have mild or moderate intellectual disabilities also suffer from dysphagia (Chadwick \& Jolliffe, 2009). The results of a study by Bastiaanse, van der Kamp, Evenhuis, \& Echteld (2014), involving people with intellectual disabilities with an average age of 61.6, showed that dysphagia was significantly associated with intellectual disability, age, and body mass index.

The signs and symptoms of dysphagia in this population may not be as obvious as they are in the typical population. Dysphagia is less self-identified, and identified by support staff/caregivers of people with intellectual disabilities (Migliore, Scoopo, \& Robey, 1999, according to Dennis, Forgeron, Morgan, \& St-Denis (2016).

Sheehan, Gandesha, Hassiotis, Gallagher, Burnell, Jones et al. (2016) cite the results of a study that included 176 hospitalized patients with intellectual disabilities. The results showed that any assessment of the swallowing status was done in only $19.3 \%$ of patients. In a study by Bastiaanse et al. (2014), the results showed that swallowing problems were not stated in the medical records of $89.5 \%$ of respondents with intellectual disabilities and dysphagia. Often, signs of dysphagia in this population are attributed to intellectual difficulty rather than a fundamental problem with the biomechanics of swallowing (Leslie et al., 2008).

Evaluation and treatment of dysphagia should be appropriate to the aetiology of the disorder and the symptoms shown by the particular patient. This requires an individualized and interdisciplinary team approach (Sheppard, 2009). The treatment of swallowing disorders is a unique challenge for professionals. Speech-language pathologist, as well as other rehabilitation specialists involved in the treatment of dysphagia, must take into account possible anatomical changes and the functional status of the swallowing mechanism in each patient.In addition, factors such as cognitive status, environment, and psychosocial aspects involved during feeding and swallowing must be considered. Therapy of swallowing disorders can begin after completing tests and detailed acquaintance with swallowing disorders in a particular patient (Murry, 2006, according to Begic, Duranovic, \& Jovanovic-Simic, 2018).

The main aim of this study was to determine the differences in the severity of dysphagia in relation to the level/degree of intellectual disabilities in persons placed in institutional accommodation.

\section{METHODS}

\section{Sample of respondents}

A total of 31 respondents with intellectual disabilities and dysphagia participated in the study, of whom 15 were male $(48 \%)$ and 16 were female $(52 \%)$. The age of the respondents ranged from 10 to 81 . 
According to the findings and recommendation of a specialist neuro-psychiatrist, the respondents were placed in the PI "Institution for the Care of Mentally Disabled Persons Drin”, Fojnica.

\section{Sample variables}

The variables used in this study can be divided into two groups:

1. Anamnestic variables: Level/Degree of intellectual disability: People with mild intellectual disabilities, People with moderate intellectual disabilities, People with severe intellectual disabilities, and People with profound intellectual disabilities.

2. Variables for dysphagia testing: Total result of direct swallowing test for semi-solids; Total result of direct swallowing test for fluid; Total result of direct swallowing test for solids; Total swallowing test result: Successful swallowing of semi-solids, liquids and solids, Successful swallowing of semi-solids and liquids and unsuccessful swallowing of solids, Successful swallowing of semi-solids and unsuccessful swallowing of liquids, Preliminary test of successful or unsuccessful swallowing of substances; Severity of dysphagia: Slight / No dysphagia, minimal risk of aspiration, Mild dysphagia with low risk of aspiration, Moderate dysphagia with risk of aspiration, Severe dysphagia with high risk of aspiration.

\section{Method of conducting research and measuring instruments}

The research was conducted in the PI "Institution for the Care of Mentally Disabled Persons Drin", in the city of Fojnica. After obtaining the consent, the manner of conducting the research was agreed in cooperation with a speech-language pathologist employed at the Institute. The sample consisted of 31 respondents with intellectual disabilities. Anamnestic data were taken from the medical documentation of the respondents. The swallowing was performed in the presence of a caregiver or medical technician, individually with each respondent, in accordance with the test propositions.

GUSS - The Gugging Swallowing Screen (Trapl, Enderle, Nowotny, Teuschl, Matz, Dachenhausen, \& Brainin, 2007) was used to detect and examine the characteristics of dysphagia in people with intellectual disabilities (translated and adapted into Bosnian). GUSS - The swallowing screening test is divided into two parts. The first part of the test refers to the preliminary test / indirect swallowing test, while the second part refers to the direct swallowing test.The preliminary test / indirect swallowing test assessed the patient's alertness, voluntary cough and / or throat clearing, ability to swallow saliva, as well as possible voice changes. The mentioned areas of the indirect swallowing test are evaluated with the answers "yes" or "no", where each of the given answers carries a certain number of points.
The maximum number of points that could be achieved on the indirect swallowing test was five points. After the indirect swallowing test, a direct swallowing test was performed. The following materials were required to perform the direct swallowing test: water, a flat tablespoon, a food thickener, and bread. The direct swallowing test assessed the ability to swallow three food consistencies, i.e. the ability to swallow semisolids, liquids and solids.Using a direct swallowing test, for all three food consistencies, the ability to swallow was first assessed, and the ability was assessed by the answers offered, which describe: that swallowing is not possible, that swallowing is delayed, or that swallowing is successful; with each given number of points.Also, for all three food consistencies, the presence or absence of involuntary cough was assessed, before, during, or after swallowing - up to three minutes after swallowing, with each of the given responses carrying a certain number of points. In addition to the above, a direct swallowing test, for all three food consistencies, assessed the presence or absence of drooling, as well as possible changes in voice before and after swallowing.Also, each of the given answers for drooling and voice changes carries a certain number of points. The maximum number of points that could be achieved on the direct swallowing test was 15 points. The total result of the swallowing test, using the indirect and direct swallowing test ranges from 0 to 20 points. An achieved score of 20 points indicates successful swallowing of semi-solid, liquid and solid textures. A score of 15 to 19 points indicates successful swallowing of semi-solid and liquid textures and unsuccessful swallowing of solid textures.A score of 10 to 14 points indicates successful swallowing of semi-solids and unsuccessful swallowing of fluids, and a score of 0 to 9 points indicates severe dysphagia with a high risk of aspiration. Based on the total result of the swallowing test, the severity of dysphagia was obtained, which can be described as: Slight / No dysphagia - minimal risk of aspiration; Mild dysphagia with low risk of aspiration; Moderate dysphagia with risk of aspiration; Severe dysphagia with high risk of aspiration.

\section{Statistical data processing}

The data were processed using the statistical program SPSS 21.0 for Windows. Statistical significance was considered for $\mathrm{p}<.05$. Basic statistical parameters were calculated: arithmetic mean, standard deviation, minimum and maximum result, and frequencies. A chi-square test was used to test the differences in the severity of dysphagia in relation to the level/degree of intellectual disability of the respondents. Correlation analysis (Spearman's correlation coefficient) was used to determine the relationship between the variables in the severity of dysphagia and the level/degree of intellectual disability. 


\section{RESULTS}

Table 1 shows the distribution of respondents with dysphagia in relation to the level/degree of intellectual disability. Out of a total of 31 respondents with dysphagia, only one respondent $(3 \%)$ was diagnosed with a mild level/degree of intellectual disability, seven respondents $(23 \%)$ with a moderate degree of intellectual disability, and 23 respondents $(74 \%)$ were diagnosed with a severe, or profound intellectual disability.

Table1. Level/degree of intellectual disability of the respondents

\begin{tabular}{lcc}
\hline Level/degree of intellectual disability & N & \% \\
\hline $\begin{array}{l}\text { Respondents with mild intellectual } \\
\text { disabilities }\end{array}$ & 1 & 3 \\
$\begin{array}{l}\text { Respondents with moderate intellectual } \\
\text { disabilities }\end{array}$ & 7 & 2 \\
$\begin{array}{l}\text { Respondents with severe, and profound } \\
\text { intellectual disabilities }\end{array}$ & 23 & 74 \\
\hline
\end{tabular}

Table 2 shows the average value of points achieved on the variable "Total result of the indirect swallowing test", which was $\mathrm{M}=5.0(\mathrm{SD}=.0)$. All respondents $(100 \%)$ achieved the maximum number of points (5) and successfully passed the indirect swallowing test, which was a prerequisite for conducting a direct swallowing test in the continuation of the study.

Table2. Total result of the indirect swallowing test

M $\overline{\mathrm{x}}$ MIN MAX

Total result of the

indirect swallowing test

$\begin{array}{llll}5.0 & .0 & 5.0 & 5.0\end{array}$

Table 3 shows the average value of the achieved points on the variable "Total result of the direct swallowing test for semi-solids" which was $\mathrm{M}=5.0(\mathrm{SD}=.0)$. All respondents $(100 \%)$ achieved the maximum number of points (5) on the direct swallowing test for semi-solids, after which the liquid swallowing test was performed.

Table 3. Total result of the direct swallowing test for semisolids

\begin{tabular}{llll} 
M & $\bar{x}$ & MIN & MAX \\
\hline
\end{tabular}

Total result of the direct

swallowing test for semi-solids

$\begin{array}{llll}5.0 & .0 & 5.0 & 5.0\end{array}$

Table 4 shows the average value of the achieved points on the "Total result of the direct swallowing test for liquids" which is $\mathrm{M}=4.5(\mathrm{SD}=.9)$. The lowest score is two points and the highest is five points. Nine respondents $(29 \%)$ scored less than a maximum of five points on the overall result of the direct swallowing test of liquids. The other 22 respondents (71\%) successfully swallow the liquid, after which they were tested for swallowing of solids.
Table 4. Total result of the direct swallowing test for liquids M SD MIN MAX

Total result of the direct swallowing test for liquids

$\begin{array}{llll}4.5 & .9 & 2.0 & 5.0\end{array}$

Table 5 shows the average value of the achieved points in the "Total result of the direct swallowing test for solids", $\mathrm{M}=1.6(\mathrm{SD}=1.6)$. The lowest achieved result is zero points, and the highest achieved result is four points.

Table5. Total result of the direct swallowing test for solids

\begin{tabular}{lcccc}
\hline & M & $\bar{x}$ & MIN & MAX \\
\hline $\begin{array}{l}\text { Total result of the direct } \\
\text { swallowing test for solids }\end{array}$ & 1.6 & 1.6 & .0 & 4.0 \\
\hline
\end{tabular}

Table 6 shows the average value of points achieved on the "Total result of the swallowing test" which is $\mathrm{M}$ $=16.1(\mathrm{SD}=2.2)$. The lowest achieved result was 12 points, and it was achieved by two respondents $(6.5 \%)$. The highest achieved result was 19 points, and it was achieved by five respondents, or $16.1 \%$. The range of points that can be achieved on the "Total result of the swallowing test" is from 0 to 20 points.

Table 6. Total result of the swallowing test

\begin{tabular}{lcccc}
\hline & M & $\overline{\mathbf{x}}$ & MIN & MAX \\
\hline $\begin{array}{l}\text { Total result of the } \\
\text { swallowing test }\end{array}$ & 16.1 & 2.2 & 12 & 19 \\
\hline
\end{tabular}

Considering the achieved total result of the swallowing test, the severity of dysphagia in the respondents was obtained. Table 7 shows the distribution of respondents in relation to the severity of dysphagia. 22 respondents $(71 \%)$ showed mild dysphagia with low risk of aspiration, and nine respondents (29\%) showed moderate dysphagia with risk of aspiration. None of the respondents had severe dysphagia.

Table 7. Severity of dysphagia of respondents

\begin{tabular}{lcc}
\hline Severity of dysphagia & N & \% \\
\hline $\begin{array}{l}\text { Mild dysphagia with low risk of } \\
\text { aspiration }\end{array}$ & 22 & 71 \\
$\begin{array}{l}\text { Moderate dysphagia with risk of } \\
\text { aspiration }\end{array}$ & 9 & 29 \\
$\begin{array}{l}\text { Severe dysphagia with high risk of } \\
\text { aspiration }\end{array}$ & 0 & 0 \\
\hline
\end{tabular}

Since there was only one respondent with a mild level/ degree of intellectual disability in the sample, due to the need for further statistical processing of the results, this respondent was included in the sample of a moderate level/degree of intellectual disability. A chi-square test was used to verify the existence of statistically significant differences in the severity of dysphagia in relation to the level/degree of intellectual disabilities of respondents. 
From Table 8 it can be noticed that in the group of people with mild and moderate intellectual disabilities, in $100 \%$ of respondents there is mild dysphagia. In contrast, in the group of people with severe, and profound intellectual disabilities, $61 \%$ of respondents have mild dysphagia and $39 \%$ of respondents have moderate dysphagia.

Table8. The severity of dysphagia in relation to the level/degree of intellectual disability

\section{Level/degree of intellectual disability}

\begin{tabular}{|c|c|c|c|c|}
\hline \multirow[b]{2}{*}{ The severity of dysphagia } & \multicolumn{2}{|c|}{$\begin{array}{l}\text { People with mild to moderate } \\
\text { intellectual disabilities }\end{array}$} & \multicolumn{2}{|c|}{$\begin{array}{l}\text { People with severe, and profound } \\
\text { intellectual disabilities }\end{array}$} \\
\hline & $\mathrm{N}$ & $\%$ & $\mathrm{~N}$ & $\%$ \\
\hline $\begin{array}{l}\text { Mild dysphagia with low risk of } \\
\text { aspiration }\end{array}$ & 8 & 100 & 14 & 61 \\
\hline $\begin{array}{l}\text { Moderate dysphagia with risk of } \\
\text { aspiration }\end{array}$ & 0 & 0 & 9 & 39 \\
\hline $\begin{array}{l}\text { Severe dysphagia with high risk of } \\
\text { aspiration }\end{array}$ & 0 & 0 & 0 & 0 \\
\hline
\end{tabular}

Table 9 shows the result of the chi-square test which showed the existence of a statistically significant difference in the severity of dysphagia in relation to the level/degree of intellectual disability $\left(\chi^{2}=4.41, \mathrm{df}=1\right.$, $\mathrm{p}=.04)$. According to the obtained results, respondents with severe, and profound intellectual disabilities have statistically significantly stronger dysphagia compared to respondents with mild, and moderate intellectual disabilities.

Table 9. Statistical significance of dysphagia severity according to the level/degree of intellectual disability

\begin{tabular}{lccc}
\hline & Value & df & p \\
\hline Pearson Chi-Square & 4.41 & 1 & .04 \\
Continuity Correction $^{b}$ & 2.72 & 1 & .10 \\
Likelihood Ratio $^{\text {Fisher's Exact Test }}$ & 6.56 & 1 & .01 \\
Linear-by-Linear Association & & & .04 \\
N of Valid Cases & 3.27 & 1 & .04 \\
\hline
\end{tabular}

It was also checked whether there was a statistically significant correlation between the level/degree of intellectual disability and the severity of dysphagia. Based on the results shown in Table 10, it is observed that the level/degree of intellectual disability has a statistically significant positive correlation with the severity of dysphagia (.38) and it can be concluded that persons with a higher level/degree of intellectual disability have more pronounced dysphagia.

Table 10. Correlations of measured factors

\begin{tabular}{ccc} 
& Level/degree of intellectual disability & The severity of dysphagia \\
\hline Level/degree of intellectual disability & 1.00 & $.38^{*}$ \\
& The severity of dysphagia & 1.00 \\
\hline${ }^{*} \mathrm{p}<.05$ &
\end{tabular}

\section{DISCUSSION}

According to the propositions of the GUSS - Screening of the swallowing test, the severity of dysphagia of the respondents was obtained by summing the achieved points on the test. The results showed that 22 respondents $(71 \%)$ had mild dysphagia and nine respondents $(29 \%)$ had moderate dysphagia. None of the respondents had severe dysphagia. Respondents with dysphagia $(\mathrm{N}=31)$ were divided into two groups according to the level/degree of intellectual disability.
One group consisted of respondents with mild, and moderate intellectual disabilities $(\mathrm{N}=8)$, and the other group consisted of respondents with severe, and profound intellectual disabilities $(\mathrm{N}=23)$. The analysis of the results showed that in the group of respondents with mild to moderate intellectual disabilities, $100 \%$ of the respondents had mild dysphagia.On the other hand, in the group of respondents with severe, and profound intellectual disabilities, $61 \%$ of respondents have mild dysphagia $(\mathrm{N}=14)$ and $39 \%$ of respondents have moderate dysphagia $(\mathrm{N}=9)$. 
The results showed the existence of a statistically significant difference in the severity of dysphagia between these two groups. According to the obtained results, it is concluded that respondents with severe, and profound intellectual disabilities have statistically significantly stronger dysphagia compared to respondents with mild, and moderate intellectual disabilities.

Numerous studies have been conducted to establish the relationship between dysphagia and the level/ degree of intellectual disability. For example, people with severe, and profound intellectual disabilities have a number of physical health problems, and research shows that the number of these problems increases with increasing the level/degree of intellectual disability (Kinnear et al., 2018, according to van Timmeren, 2019). Also, due to receiving a larger amount of prescribed medications, people with severe, and profound intellectual disabilities are often exposed to the side effects of such drugs (van Timmeren, 2019). Intellectual, motor, and sensory difficulties reinforce each other so that people with severe, and profound intellectual disabilities have less ability to compensate for the difficulties present (Kiestra, 2005, according to van Timmeren, 2019) and thus swallowing disorders. Decreased attention, reduced ability to compensate for swallowing disorders along with the listed primary, congenital, difficulties and those arising from them, lead to more pronounced dysphagia in persons with severe, and profound intellectual disabilities.

In a study by Matson, Fodstad and Boisjoli (2008), 14 of $23(60.9 \%)$ STEP test items (Norms and patterns of feeding problems for the screening tool of feeding problems) were present in respondents with profound intellectual disabilities versus respondents with mild to severe intellectual disabilities. Some of these items include the following: does not show the ability to chew (13.2\% profound, versus $3.4 \%$ mild to severe intellectual disability); chokes on food (3.5\% profound vs. $2.3 \%$ mild to severe intellectual disability); does not show the possibility of swallowing $(3.8 \%$ profound vs. $1.1 \%$ mild to severe intellectual disability); swallowing without effective chewing $(5.7 \%$ profound vs. $3.4 \%$ mild to severe intellectual disability); special positioning for feeding is required $(26.1 \%$ profound vs. $3.4 \%$ mild to severe intellectual disability). Another factor that has had a significant impact on dysphagia in people with intellectual disabilities is the presence of cerebral palsy. Calis, Veugelers, Sheppard, Tibboel, Evenhuis and Penning (2008), on a sample of 166 children with cerebral palsy and intellectual disabilities, received a result showing that only $1 \%$ of respondents did not have dysphagia, $8 \%$ had mild dysphagia, $76 \%$ moderate to severe dysphagia, and $15 \%$ profound dysphagia. The same study also showed an association between the severity of dysphagia and the severity of motor difficulties. An association between the level/degree of intellectual disabilities and dysphagia was shown in children with cerebral palsy in the study conducted by Waterman et al. (1992, according to Robertson, Chadwick, Baines, Emerson,
\& Hatton, 2017). According to their results, dysphagia was present in $12.5 \%$ of children with mild, or moderate intellectual disabilities, in $40 \%$ of children with severe intellectual disabilities, and in $44 \%$ of children with profound intellectual disabilities.

Thacker, Abdelnoor, Anderson, White and Hollins (2008) examined the incidence of suffocation episodes in people with intellectual disabilities, which may be a direct consequence of dysphagia. The results of the study showed that $42 \%$ of respondents with intellectual disabilities had one or more episodes of suffocation. The occurrence of suffocation episodes, according to the results of their research, was significantly more common in people with a higher level/degree of intellectual disabilities, with Down syndrome, with incomplete dentition and in people who use psychotropic drugs more often.

\section{CONCLUSION}

In the conducted study, the level/degree of intellectual disability had a significant impact on the severity of dysphagia. The largest percentage of respondents with intellectual disabilities (71\%) had mild dysphagia with a low risk of aspiration. Moderate dysphagia with a risk of aspiration was present in $29 \%$ of respondents with intellectual disabilities.Severe dysphagia with a high risk of aspiration was not reported in any of the respondents in this study. According to the level/degree of intellectual disability, statistically significantly stronger dysphagia was experienced by persons with severe, and profound intellectual disabilities compared to persons with mild, and moderate intellectual disabilities.In the group of people with mild, and moderate intellectual disabilities, $100 \%$ of respondents had mild dysphagia, in contrast to the group of respondents with severe, and profound intellectual disabilities where $39 \%$ of respondents had moderate dysphagia and $61 \%$ of respondents had mild dysphagia. The severity of dysphagia, and the level/ degree of intellectual disabilities have a statistically significant positive correlation and it can be concluded that people with a higher level/degree of intellectual disability have more pronounced dysphagia.

Given all the results obtained in this study, it can be concluded that dysphagia is a significant problem in the population of people with intellectual disabilities, especially in those with severe, and profound intellectual disabilities. It is important to point out the importance of early detection, monitoring and treatment of dysphagia in this population, in order to reduce the possible progression of dysphagia and reduce its complications. 


\section{REFERENCES}

American Psychiatric Association. (2013). Diagnostic and Statistical Manual of Mental Disorders, Fifth Edition. Arlington, VA. American Psychiatric Publishing, Inc.

Bastiaanse, L., van der Kamp, A., Evenhuis, H., \& Echteld, M. (2014). Dysphagia in older people with intellectual disabilities: results of the HA-ID study. In: Bastiaanse, L. (2014). (Ed). Nutrition, Nutritional State and Related Conditions in Older Adults with Intellectual Disabilities. Rotterdam: Erasmus Universiteit Rotterdam, 57-68.

Begic, L., Duranovic, M., \& Jovanovic-Simic, N. (2018). Osnove disfagija. Foca. Univerzitet u Istocnom Sarajevu: Medicinski fakultet Foca.

Begic, L., \& Salihovic, N. (2018). Disfagija. Tuzla: MAXI PLUS d.o.o.

Calis, E.A.C., Veugelers, R., Sheppard, J.J., Tibboel, D., Evenhuis, H.M., \& Penning, C. (2008). Dysphagia in children with severe generalized cerebral palsy and intellectual disability. Developmental Medicine \& Child Neurology, 50, 625-630. doi:10.1111/j.1469-8749.2008.03047.x

Chadwick, D.D., Jolliffe, J., \& Goldbart, J. (2003). Caregiver knowledge and barriers to their compliance with dysphagia management strategies. Proceedings of the $5^{\text {th }}$ European CPLOL Congress, Evidence Based Practice: A challenge for Speech and Language Therapists, Heriott Watt Conference Centre. Edinburgh, UK, $5^{\text {th }}-7^{\text {th }}$ Sept, 2003. https://www. researchgate.net/publication/272504048 Dysphagia management_for_adults_with_learning_disabilities _ Caregiver knowledge and barriers to their adherence

Chadwick, D.D., \& Jolliffe, J. (2009). A descriptive investigation of dysphagia in adults with intellectual disabilities. Journal of Intellectual Disability Research, 53(1), 29-43. doi: 10.1111/j.1365-2788.2008.01115.x.

Dennis, S.,Forgeron, S., Morgan, H., \& St-Denis, J. (2016). Special Considerations for Speech Language Pathologists Serving People with Intellectual Disabilities. A supplementary resource to augment existing practice guidelines. http:// www.community-networks.ca/wp-content/uploads/2015/07/ SLP-Guidelines-Final-March-9-2016.pdf

Kolundzic, Z. (2012). Disfagija - simptom bolesti ili stanja. Znanstveno-strucni simpozij Logopedija: jucer, danas, sutra. Zagreb, Hrvatska, 9.11.2012. file:///C:/Users/korisniks/ Downloads/disfagija-simptom-bolesti-ili-stanja-kolundzic. pdf
Leslie, P., Crawford, H., \& Wilkinson, H. (2008). People with a Learning Disability and Dysphagia: A Cinderella Population? Dysphagia, 24(1), 103-104.DOI: 10.1007/s00455-008-91534.

Lindsay, P. (2011). Care of the Adult with Intellectual Disability in Primary Care. London, New York. Radcliffe Publishing.

Matson, J.L., Fodstad, J.C., \& Boisjol, J.A. (2008). Cutoff scores, norms and patterns of feeding problems for the Screening Tool of fEeding Problems (STEP) for adults with intellectual disabilities. Research in Developmental Disabilities, 29(4),363-372.doi: 10.1016/j.ridd.2007.06.001.

Robertson, J., Chadwick, D., Baines, S., Emerson, E. \& Hatton, C. (2017). Prevalence of Dysphagia in People With Intellectual Disability: A Systematic Review. Intellectual and Developmental Disabilities, 55(6), 377-391.doi: 10.1352/1934-9556-55.6.377.

Sheppard, J.J. (1991). Managing Dysphagia in Mentally Retarded Adults. Dysphagia, 6, 83-87.

Sheppard, J.J. (2006). Developmental Disability and Swallowing Disorders in Adults. In: Cichero, J.A., Murdoch, B.E. (2006). (Eds). Dysphagia: Foundation, Theory and Practice. Chichester.Chichester, New York, Weinheim, Brisbane, Toronto, Singapore. John Wiley \& Sons, Ltd., 299-318.

Sheppard, J.J. (2009). Intellectual and Developmental Disability. In: Jones, H.N., \& Rosenbek, J.C. (2009). (Eds). Dysphagia in Rare Conditions: An Encyclopedia. San Diego. Plural Publishing Inc., 295-308.

Thacker, A., Abdelnoor, A., Anderson, C., White, S., \& Hollins, S. (2008). Indicators of choking risk in adults with learning disabilities: A questionnaire survey and interview study. Disability and Rehabilitation, 30(15), 1131-1138.doi: 10.1080/09638280701461625.

Trapl, M., Enderle, P., Nowotny, M., Teuschl, Y., Matz, K. Dachenhausen, A., Brainin, M. (2007). Dysphagia Bedside Screening for Acute-Stroke Patients The Gugging Swallowing Screen. Stroke, 38, 2948-52. doi: 10.1161/ STROKEAHA.107.483933.

van Timmeren,E.A. (2019). Physical health in adults with severe or profound intellectual and motor disabilities. Rijksuniversiteit Groningen. 